\title{
Interactions of a small molecule configuration mimetic the interactions found in $\alpha$-synuclein aggregation
}

\author{
Marco A. Saraiva ${ }^{1}$ and M. Helena Florêncio ${ }^{2}$ \\ ${ }^{1}$ Universidade de Lisboa Centro de Quimica Estrutural \\ ${ }^{2}$ Universidade de Lisboa Departamento de Quimica e Bioquimica
}

September 24, 2021

\begin{abstract}
Parkinson's disease (PD) is an increasingly prevalent and currently incurable neurodegenerative disorder. The aggregation of the amyloid disordered protein $\alpha$-synuclein (Syn) has been implicated in the development of PD. Syn aggregation has been widely investigated but information concerning the conformational alterations in the diverse protein aggregated species at the molecular level is still scarce. To address this issue, it was here developed a comparative study involving the known parent $N \alpha$-acetyl-L-tyrosinamide (NAYA) compound and the Syn protein by using spectroscopic techniques. At least two different configurations of the NAYA compound were found to exist in solution. One configuration, known as the NAYA closed shape configuration, involves an amide intramolecular hydrogen-bonded interaction and was found to be a model interaction for the hydrophilic core of $\beta$-sheets, which are the most common conformational alteration found in Syn aggregated species. Since the spectroscopic techniques used herein also differentiate between tyrosyl and peptide bond groups and both NAYA and Syn possess such groups it was possible to assign these groups interactions in the $\beta$-sheets formed. This study retrieves the importance of using model compounds with spectroscopic characteristics similar to those found in proteins to access the complex interactions network existing in the amyloid aggregated species.
\end{abstract}

\section{Hosted file}

Manuscript_Proteins.docx available at https://authorea.com/users/435878/articles/538606interactions-of-a-small-molecule-configuration-mimetic-the-interactions-found-in-\%CE\%B1synuclein-aggregation 